\title{
OPEN Specific domain $V$ reduction of beta-2-glycoprotein I induces protein flexibility and alters pathogenic antibody binding
}

Ina Buchholz ${ }^{1,2,11}$, Thomas McDonnell 3,11 , Peter Nestler ${ }^{4}$, Sudarat Tharad ${ }^{5}$, Martin Kulke ${ }^{1}$, Anna Radziszewska ${ }^{6,7}$, Vera M. Ripoll ${ }^{7}$, Frank Schmidtt ${ }^{8,9}$, Elke Hammer ${ }^{8,10}$, Jose L. Toca-Herrera ${ }^{5}$, Anisur Rahman ${ }^{7 凶}$ \& Mihaela Delcea ${ }^{1,2,10 \bowtie}$

Beta-2-glycoprotein I ( $\beta 2 \mathrm{GPI}$ ) is a blood protein and the major antigen in the autoimmune disorder antiphospholipid syndrome (APS). $\beta 2 \mathrm{GPI}$ exists mainly in closed or open conformations and comprises of 11 disulfides distributed across five domains. The terminal Cys288/Cys326 disulfide bond at domain $\mathrm{V}$ has been associated with different cysteine redox states. The role of this disulfide bond in conformational dynamics of this protein has not been investigated so far. Here, we report on the enzymatic driven reduction by thioredoxin-1 (recycled by Tris(2-carboxyethyl)phosphine; TCEP) of $\beta 2 \mathrm{GPI}$. Specific reduction was demonstrated by Western blot and mass spectrometry analyses confirming majority targeting to the fifth domain of $\beta 2 \mathrm{GPI}$. Atomic force microscopy images suggested that reduced $\beta 2 \mathrm{GPI}$ shows a slightly higher proportion of open conformation and is more flexible compared to the untreated protein as confirmed by modelling studies. We have determined a strong increase in the binding of pathogenic APS autoantibodies to reduced $\beta 2 \mathrm{GPI}$ as demonstrated by ELISA. Our study is relevant for understanding the effect of $\beta 2 \mathrm{GPI}$ reduction on the protein structure and its implications for antibody binding in APS patients.

Beta-2 Glycoprotein I ( $\beta 2 \mathrm{GPI})$ is a five-domain glycoprotein is comprised of 326 amino acids ${ }^{1}$. The C-terminal Domain V (DV) is responsible for the ability of $\beta 2$ GPI to bind to cell surfaces. DIII and DIV are highly glycosylated with 4 or 5 glycan sites ${ }^{2-4}$. The beta-sheet rich domains are separated by flexible random coil regions that allow the protein to exhibit two major conformational states; the closed (circular) conformation and open (linear) conformation. The circular form was visualized by Agar et al. ${ }^{5}$, while the linear conformation was shown by X-ray crystal structure analysis ${ }^{1,6}$. A potential S-shaped conformation, which may represent an intermediate form, has been hypothesized ${ }^{7,8}$.

$\beta 2 \mathrm{GPI}$ is the major antigen in the autoimmune disorder antiphospholipid syndrome (APS) ${ }^{9-13}$. APS has an incidence of 2 per 100,000 people per year ${ }^{14}$ and is a leading cause of recurrent miscarriage and of strokes in patients under 50 years old. The major epitopic region of $\beta 2$ GPI, R39-G43, resides in DI ${ }^{15-19}$. Antibodies to this region have been shown to be pathogenic in mouse models and correlate well with thrombosis and pregnancy morbidity in patients ${ }^{13,17,19-21}$. It is hypothesized that in the circular form, this region in DI is hidden, giving the effect of a cryptic epitope $e^{5,22}$.

$\beta 2$ GPI has a number of physiological functions in the healthy body, which have been recently reviewed ${ }^{23}$, including both up- and down-regulation of the complement and coagulation cascades, a role in LPS scavenging ${ }^{24}$ and in prevention of melanoma spread ${ }^{25}$. The ability to act within two cascades in opposing manners is relatively unique to $\beta 2 \mathrm{GPI}$.

${ }^{1}$ Institute of Biochemistry, University of Greifswald, Greifswald, Germany. ${ }^{2}$ ZIK HIKE, University of Greifswald, Greifswald, Germany. ${ }^{3}$ Division of Biochemical Engineering, Bernard Katz Institute, University College London, London, UK. ${ }^{4}$ Institute of Physics, University of Greifswald, Greifswald, Germany. ${ }^{5}$ Institute for Biophysics, University of Natural Resources and Life Sciences Vienna, Vienna, Austria. ${ }^{6}$ Centre for Adolescent Rheumatology Versus Arthritis at UCL, UCLH, GOSH, London, UK. 'Division of Medicine, Centre for Rheumatology, University College London, London, UK. ${ }^{8}$ Interfaculty Institute for Genetics and Functional Genomics, University of Greifswald, Greifswald, Germany. ${ }^{9}$ Proteomics Core, Weill Cornell Medicine-Qatar, Doha, Qatar. ${ }^{10}$ DZHK (German Centre for Cardiovascular Research), Partner Site Greifswald, Greifswald, Germany. ${ }^{11}$ These authors contributed equally: Ina Buchholz and Thomas McDonnell. ${ }^{\circledR}$ email: anisur.rahman@ucl.ac.uk; delceam@uni-greifswald.de 


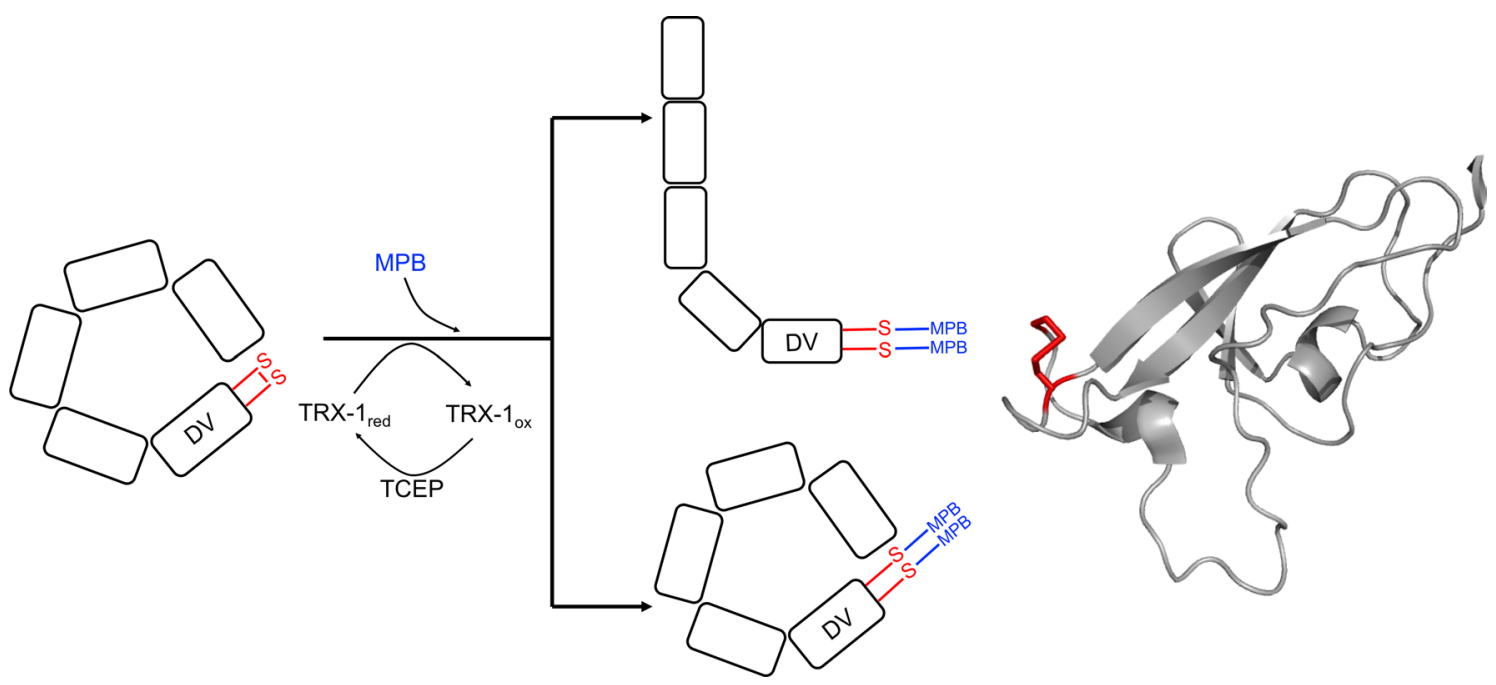

Figure 1. Left panel: schematic of the hypothesised reaction on circular $\beta 2 \mathrm{GPI}$, highlighting the intact disulfide bond in the fifth domain (DV). The protein undergoes selective, enzymatic driven reduction by TRX-1 (recycled by TCEP) and liberates the two thiols, which are subsequently labelled by MBP to avoid re-oxidation. All reaction steps were performed under argon atmosphere at $25^{\circ} \mathrm{C}$ in $\mathrm{HBS}$ buffer $(\mathrm{pH} 7.4)$ with mild shaking. $50 \mu \mathrm{M}$ TRX-1 was reduced by $50 \mu \mathrm{M}$ TCEP in a total volume of $100 \mu \mathrm{L}$ HBS buffer for $1 \mathrm{~h} .900 \mu \mathrm{L}$ HBS buffer and $0.2 \mu \mathrm{M} \beta 2 \mathrm{GPI}$ were added and incubated for $1 \mathrm{~h}$. $15 \mu \mathrm{M}$ MPB were added for $15 \mathrm{~min}$ to label free thiols. Finally, $200 \mu \mathrm{M}$ reduced GSH was used to quench unbound MPB (15 min). Right panel: C-terminal, fifth domain (DV) of $\beta 2$ GPI with the disulfide Cys288/Cys326 highlighted in red.

Although the role of post-translational modifications in altering the structural conformation of $\beta 2$ GPI has been investigated ${ }^{26,27}$, the potential relationship between alternative structures of $\beta 2 \mathrm{GPI}$ and the different functions of the molecule is not understood fully.

In this paper, we examine the effect of disulfide reduction on the structure of $\beta 2 \mathrm{GPI}$. All five domains of $\beta 2 \mathrm{GPI}$ contain two structural disulfides with little surface exposure. DV alone has a third disulfide (Cys288/Cys326) which is allosteric, generally associated with a structural change of proteins ${ }^{28}$. As shown by Ioannou et al. ${ }^{29}$, 32 GPI includes different cysteine redox states of the Cys288/Cys326 disulfide bond under pseudo-physiological conditions $^{27}$. Protein redox states have also been shown to be of interest in the pathogenesis of APS ${ }^{30,31}$, and in the production of pathogenic anti- $\beta 2$ GPI antibodies ${ }^{31}$. Studies looking at the terminal disulfide in DV have been conducted showing an association with the production of anti-DI antibodies ${ }^{32-34}$. However, the effects of reduction on the structural conformation of $\beta 2 \mathrm{GPI}$ have not been fully elucidated. This study aims to fully characterize by spectroscopic and imaging techniques the effect of specific reduction of Cys288/Cys326 on the structure of $\beta 2 \mathrm{GPI}$, and its implications for binding of antibodies from patients with APS.

\section{Results}

Enzymatic reduction of $\beta 2 \mathrm{GPI}$ mainly targets the Cys288/Cys326 disulfide bond. Specific reduction of $\beta 2$ GPI was carried out by TRX-1 as described in the "Methods" section (Fig. 1) and purification was achieved by SEC. To investigate the redox state of the protein, an Ellman's reagent assay was used to quantify free thiols within untreated $\beta 2$ GPI (Supplementary Fig. S1). Although $\beta 2$ GPI has 22 cysteine residues, untreated protein gave a ratio of $0.19 \pm 0.02: 1$ thiols per protein, indicating that the majority of protein's thiols are in oxidized state.

Reaction mixture was purified and uniform enzymatic reduction and homogenous MPB labelling were confirmed via SDS-PAGE and streptavidin Western blot (Fig. 2A, lanes 2 and 3). Lack of thiols in the untreated protein was further confirmed as no band was identified by streptavidin Western blot (Fig. 2A, lane 4). Purity of reduced species was confirmed by SDS-PAGE (Fig. 2A, lane 5). The biotin quantification assay detected a ratio of $1.0 \pm 0.3$ biotin molecules per $\beta 2 \mathrm{GPI}$ molecule, which suggested MPB labelling only on a single thiol.

Specific labelling was confirmed by LC-MS/MS analysis. A total of 10 peptides were identified to contain MPB (Supplementary Table S1) when the protein was digested with trypsin in the native state. All sequence numbers refer to those of the mature sequence as listed in PDB $1 \mathrm{c} 1 \mathrm{z}$, this corresponds to amino acids 20-345 in the immature sequence as stated in Uniprot P02749 and thus, excludes the signaling peptide 1-19. The terminal peptide (peptide 10) containing Cys326 showed the highest response for MPB labelling (Fig. 2B). The peptides with the highest responses were mapped onto a protein model (pdb-ID: 1c1z): peptide 9 (cyan) and peptide 10 (red) contain matching thiols liberated from disulfide bond Cys288/Cys326 (Fig. 2C).

$\beta 2$ GPI reduction initiates a structural change. Statistical analysis of the conformation of $\beta 2$ GPI was carried out based on AFM imaging as previously published ${ }^{26}$. Images of both reduced and untreated $\beta 2$ GPI (Fig. 3A,B) were analysed for particle shape, which is represented by cartoon images (Fig. 3C). 
A

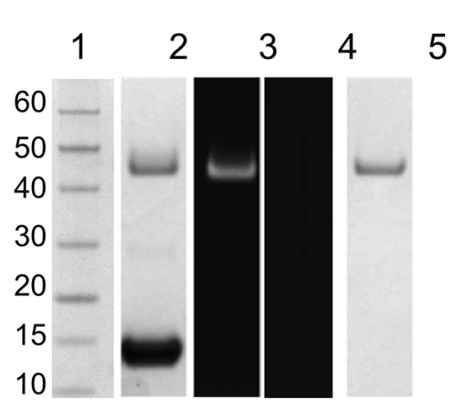

B

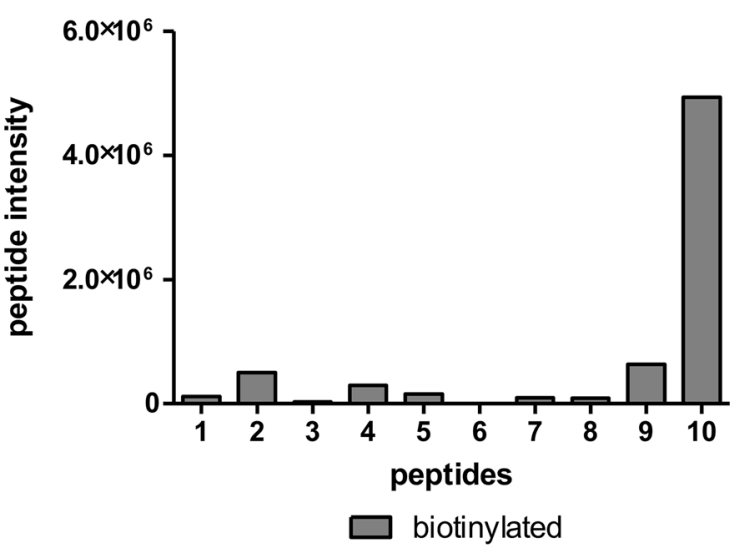

C

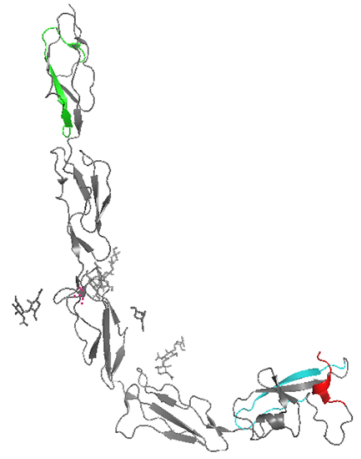

Figure 2. (A) SDS-PAGE and Western blot analyses (full-length gels and blots with multiple exposures are included in Supplementary Figs. S2-S4): lane 1-Marker, lane 2-the reaction mixture with $\beta 2$ GPI and then TRX (labelled), lane 3-detection of $\beta 2$ GPI using a specific streptavidin HRP enzyme by Western blot, lane 4-unlabelled $\beta 2$ GPI in a streptavidin specific Western blot, lane 5-sample purified by SEC, yielding highly pure labelled protein. (B) Peptide intensities obtained by LC-MS/MS analysis from labelled $\beta 2$ GPI. (C) Crystal model with the three highest responding peptides highlighted: peptide 10 (sequence $318-326$ in DV) in red, peptide 9 (sequence 287-305 in DV) in cyan, peptide 2 (sequence 20-39 in DI) in green. Peptide 287-305 consists of the disulfide partner for peptide TDASDVKPC, suggesting that specific labelling has been achieved.

A

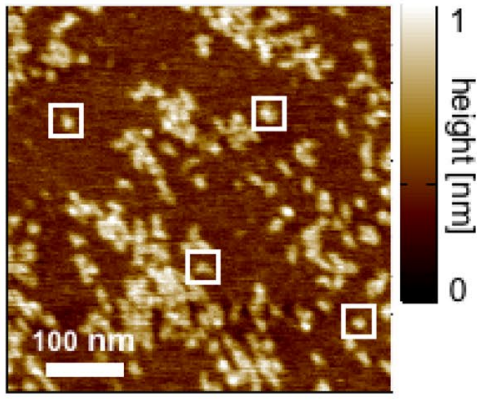

B

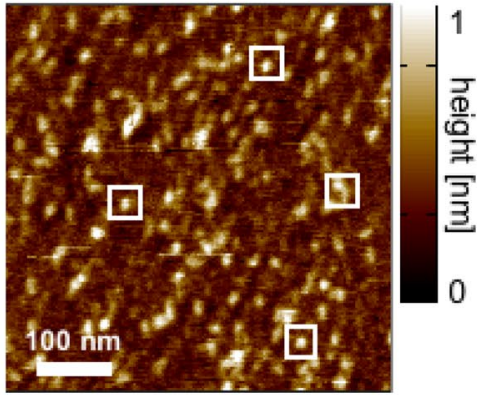

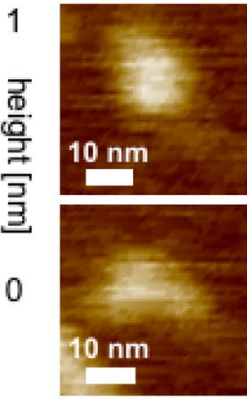

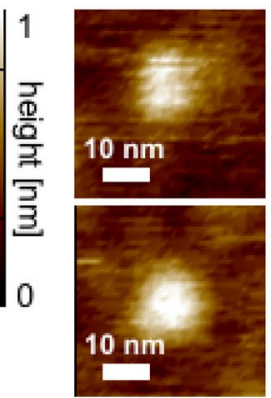

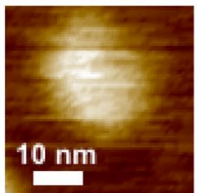
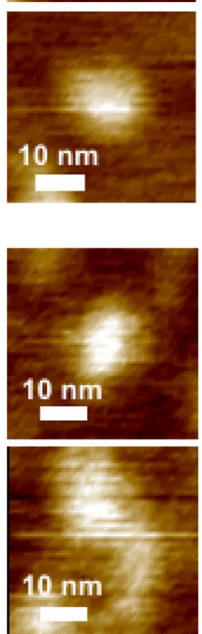

C
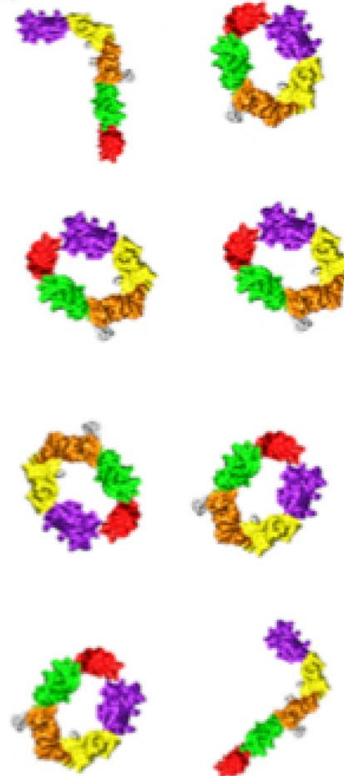
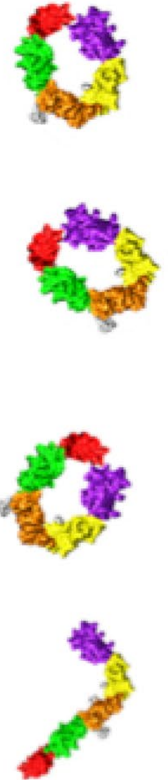

D

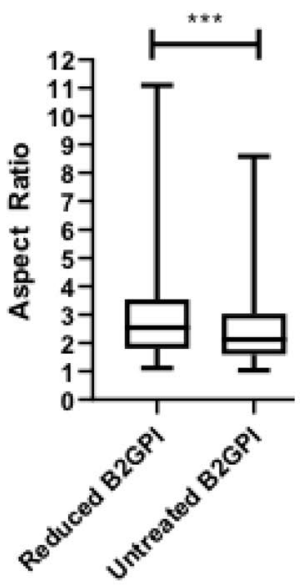

Figure 3. AFM imaging of $\beta 2$ GPI. Untreated (A) and reduced (B) $\beta 2$ GPI with examples of particle structure highlighted. (C) Surface models of $\beta 2$ GPI in the suggested conformation of the structures highlighted in (A) and (B). (D) Distribution of aspect ratios $R$ with the minimum and maximum values (whiskers) plotted with the median, this includes three independent replicates of reduced $\beta 2 \mathrm{GPI}$ and two replicates (with two technical replicates) of untreated $\beta 2$ GPI. In total, 351 and 352 molecules were analysed, respectively. A higher mean aspect ratio for reduced $\beta 2$ GPI (2.97) compared to untreated $\beta 2$ GPI (2.58) and a significantly increased percentage of open particles ( $35 \%$ vs $25 \%$ ) were found ( $p=0.0003$ ). This suggests that reduction allows $\beta 2$ GPI to be more flexible, which is best reflected as a wider upper distribution of structures with more structures falling above an aspect ratio of 5 ( $10.5 \%$ vs $6.3 \%$ reduced and untreated, respectively). 
Reduced protein

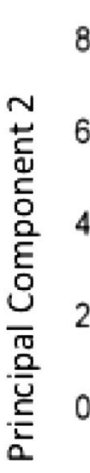

8

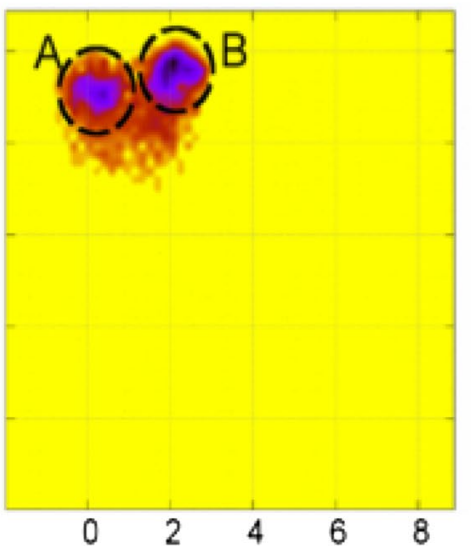

Untreated protein

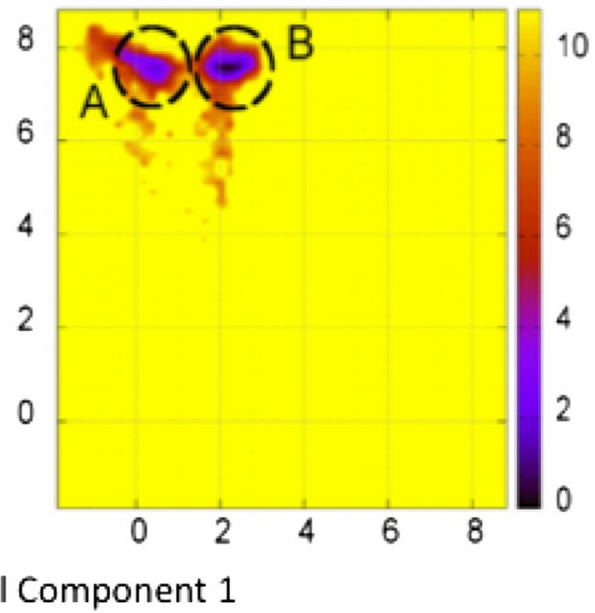

Figure 4. Principal Component Analysis were generated with an in-house code and plotted with GnuPlot 5.4 software (http://www.gnuplot.info) as energy histograms: reduced (left) and untreated (right) $\beta 2 \mathrm{GPI}$. The degree of variation is greater in the reduced protein, with larger areas of red seen, whilst the oxidized protein remains fairly stable with smaller areas of red. This suggests greater flexibility in the reduced protein.

As defined in the methods section, aspect ratio $R$ (particle length/width) above 3 was considered to be open $\beta 2$ GPI, whilst a value lower than 3 was defined as "closed" $\beta 2 G^{26}$. For both untreated and reduced $\beta 2$ GPI, the majority of molecules were closed, but reduced $\beta 2$ GPI showed a significantly higher proportion of proteins with an aspect ratio $>3$ compared to the untreated protein $(\mathrm{p}=0.0003)$.

Although mean aspect ratio was similar between untreated and reduced, the spread of values was higher for reduced $\beta 2$ GPI (Fig. 3D) with increased variance and standard deviation as illustrated in Supplementary Table S2.

We further complemented the AFM measurements with DLS protein size determination analyses which showed a significant shift in hydrodynamic size (Supplementary Fig. S5A). Untreated $\beta 2$ GPI (black) had a hydrodynamic diameter $\left(D_{H}\right)$ of $13.1 \pm 0.7 \mathrm{~nm}$ in agreement with previous size determinations ${ }^{26}$. For reduced $\beta 2$ GPI species (red), a significantly smaller $\mathrm{D}_{\mathrm{H}}$ of $10.1 \pm 1.1 \mathrm{~nm}$ was found, which was previously associated with a protein conformational change $\mathrm{e}^{26,35}$.

CD data of untreated (black) and reduced (red) $\beta 2$ GPI showed spectra limited to wavelength above $216 \mathrm{~nm}$ due to interfering effects of the biotin function and the HBS buffer (Supplementary Fig. S5B). As can be observed, the $\mathrm{CD}$ peaks of reduced $\beta 2 \mathrm{GPI}$ (red) remain largely within the standard deviations (green and blue) for untreated $\beta 2 \mathrm{GPI}$. The slight differences between the two $\beta 2 \mathrm{GPI}$ species suggested that labelling did not affect the secondary structure and the process has not led to denaturation of $\beta 2 \mathrm{GPI}$.

Modelling studies confirm enhanced flexibility of reduced $\beta 2 \mathrm{GPI}$ without major change in secondary or tertiary structure. A structural model of $\beta 2 \mathrm{GPI}$ was generated with a reduced disulfide in DV. Later assembly of a closed $\beta 2$ GPI conformation based on affinity analysis was carried out and supplemented with extended carbohydrate chains which stabilized within 70 ns of equilibration. Molecular dynamics simulation (MDS) of reduced $v s$ oxidized $\beta 2$ GPI was undertaken for $350 \mathrm{~ns}$ and no significant change was seen in tertiary and secondary structure. However, maximal root mean square deviation (RMSD) values between two major groups varied greatly (Fig. 4, spot A and spot B). Reduced 32 GPI showed a maximal RMSD of $\sim 15$ with a range of $4-7$, whilst oxidized $\beta 2$ GPI had a much lower range and a maximal value of just 3.6. Nevertheless, $A$ and $\mathrm{B}$ are structurally only minorly different with the main deviations being located at the two loop regions at the tip of DV. For both $\beta 2$ GPI forms, structure $A$ is the main population with A:B being 2:1, while the energy barrier between the spots is $1.6 \mathrm{~kJ} / \mathrm{mol}$ and $1.2 \mathrm{~kJ} / \mathrm{mol}$ for reduced and oxidized $\beta 2 \mathrm{GPI}$, respectively. Both energy barriers are easily overcome by thermal energy making the transition between A and B fast, which suggests an increase in flexibility, with the largest deviations being within DV for reduced $\beta 2$ GPI. This was confirmed by dihedral Principal Component Analysis ${ }^{36}$ which demonstrated increased variation in reduced $\beta 2$ GPI (Fig. 4).

Reduction of $\beta 2 \mathrm{GPI}$ increases binding of IgG anti-DI samples from patients with APS. Polyclonal IgG anti-DI antibodies were purified from four triple-positive APS patients, whose characteristics can be seen in Table 1.

A control experiment was carried out to exclude the possibility that the secondary $\beta 2 \mathrm{GPI}$-specific antibody showed preferential binding to reduced $\beta 2$ GPI. Figure 5B shows the results of the control ELISA, where the plate was coated directly with both $\beta 2$ GPI species and the $\beta 2$ GPI-specific secondary antibody was added. No difference was seen between binding of secondary antibody to reduced and untreated $\beta 2 \mathrm{GPI}$, indicating that the difference in Fig. $5 \mathrm{~A}$ must be due to differential binding of $\beta 2 \mathrm{GPI}$ species to the polyclonal patient-derived IgG anti-DI on the plate. 


\begin{tabular}{|c|c|c|c|c|c|c|c|c|c|}
\hline Patient no & Age & Sex & Type of APS & Thrombotic events & Pregnancy morbidity & LA-positive & aCL & Anti-beta2GPI & Anti-DI \\
\hline 1 & 53 & Male & PAPS & 1 Arterial & Not applicable & Yes & $>100$ & 86 & 26 \\
\hline 2 & 57 & Female & SLE Associated & Multiple Venous & $\begin{array}{l}\text { One termination of pregnancy for medical } \\
\text { reasons }\end{array}$ & Yes & $>100$ & 85 & 89 \\
\hline 3 & 74 & Male & PAPS & Multiple Venous and Arterial & Not applicable & Yes & $>100$ & $>100$ & $>100$ \\
\hline 4 & 37 & Female & SLE Associated & 1 Arterial & $\begin{array}{l}\text { One first trimester loss, one premature } \\
\text { delivery } 29 / 52 \text { due to placental throm- } \\
\text { bosis }\end{array}$ & Yes & $>100$ & $>100$ & $>100$ \\
\hline
\end{tabular}

Table 1. Patient information for the study. Demographic and clinical characteristics, as well as antibody levels are given. PAPS primary antiphospholipid syndrome, SLE systemic lupus erythematosus, $L A$ lupus anticoagulant, $a C L$ anti cardiolipin antibodies. Triple positivity (positive tests 12 weeks apart for anti-

Cardiolipin (aCL), anti-Beta-2-Glycoprotein I (aß2GPI) and Lupus Anticoagulant) is associated with a more severe phenotype for APS patients ${ }^{36,37}$. These polyclonal IgG samples were tested for binding to untreated and reduced $\beta 2$ GPI. Significantly higher binding was seen with reduced protein at all concentrations (Fig. 5A). A dose-dependent response was observed with a maximal concentration of $5 \mu \mathrm{g} / \mathrm{mL}$ reduced $\beta 2 \mathrm{GPI}$ giving an OD of $\sim 1$.

A

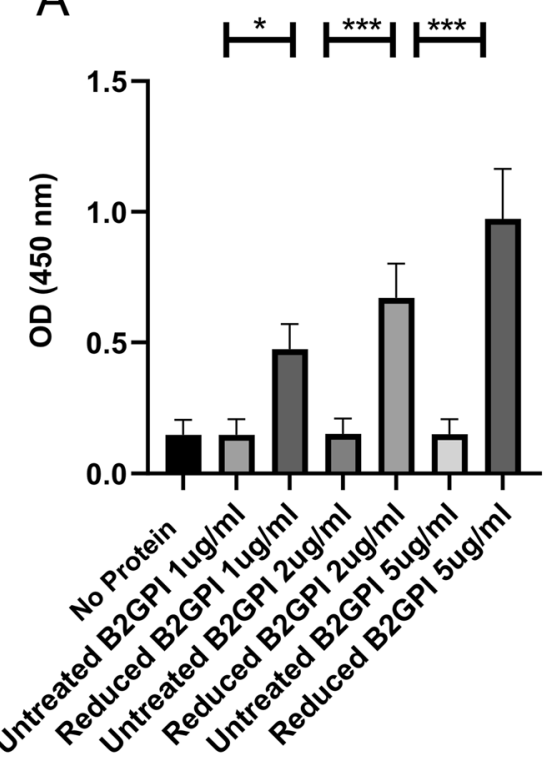

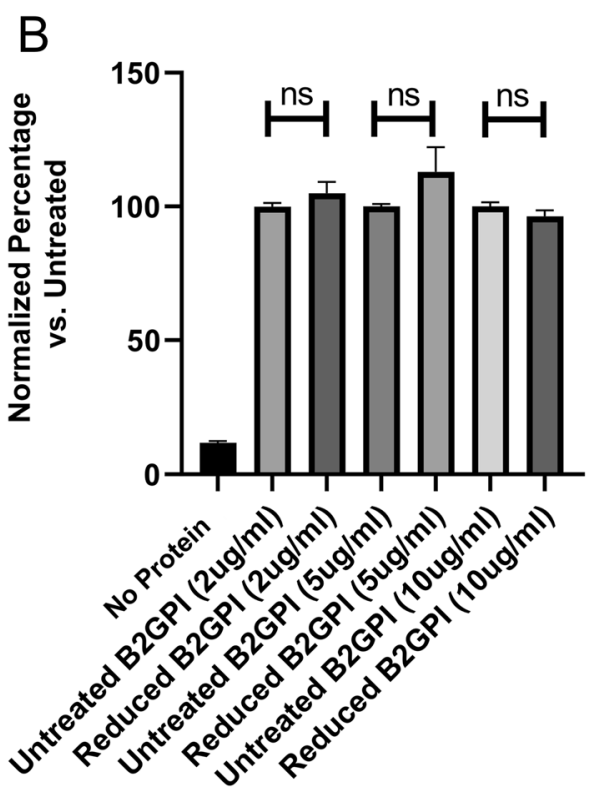

Figure 5. Assessment of antigenicity of reduced $\beta 2$ GPI. Antibodies to DI of $\beta 2$ GPI were purified from serum of APS patients using immunoprecipitation with a proprietary tagged DI molecule on a nickel bead. (A) Purified antibodies were coated on a maxisorb plate and incubated with either reduced or untreated $\beta 2 \mathrm{GPI}$. Detection occured via a secondary peroxidase-conjugated anti- $\beta 2$ GPI antibody and OD was read at $450 \mathrm{~nm}$. (B) Data from the control ELISA with either reduced or untreated $\beta 2$ GPI coated on a maxisorb plate and detected with the same detection system as previously described. As it can be seen, the secondary antibody does not favour detection of the reduced form. The data in Panel B has been normalized, so the effect of untreated $\beta 2 \mathrm{GPI}$ is considered $100 \%$.

\section{Discussion}

Our results suggests that, although there is a dramatic increase in binding of anti-DI antibodies from APS patients to reduced $\beta 2 \mathrm{GPI}$, the structural alteration upon reduction may be more subtle than first hypothesized. This may be explained by an increased flexibility of the molecule, rather than a traditional shift of conformation from closed to open.

Our data challenges several paradigms in the field. It has been assumed by other studies that $\beta 2$ GPI upon purification becomes oxidized and linear. Here, we show that the majority of purified, untreated $\beta 2 \mathrm{GPI}$ is oxidized but also circular. A second assumption was that antibodies from patients would bind better to oxidized $\beta 2 \mathrm{GPI}$ due to a structural shift to the open form. However, we found that anti-DI antibodies from patients with APS actually bind more strongly to reduced $\beta 2 \mathrm{GPI}$.

Ioannou et al. showed that it was possible to label thiols of $\beta 2 \mathrm{GPI}$ in plasma ${ }^{15,34}$, and proposed that a larger proportion of circulating $\beta 2 \mathrm{GPI}$ is in the free-thiol form ${ }^{38}$ in healthy controls than in patients with $\mathrm{APS}^{38}$. Their suggestion was that increased oxidized $\beta 2$ GPI in these patients exposed DI favouring production of anti-DI antibodies. Raimondo et al. ${ }^{32}$ reported increased levels of reduced $\beta 2$ GPI in the serum of anti-DI negative compared to anti-DI positive patients. However, Raimondo and co-workers used an indirect method in which the amount 
of reduced $\beta 2 \mathrm{GPI}$ was expressed compared to a pooled healthy control, without correction for increased levels of total $\beta 2$ GPI in APS patients.

Although it had been firmly established in the literature that circulating $\beta 2$ GPI was in the circular conformation $^{5}$, a recent publication by Ruben et al. ${ }^{39}$ has presented evidence to the contrary. Importantly, the work by Ruben et al. uses a number of biophysical techniques which have not previously been applied to $\beta 2$ GPI including SAXS and FRET, whilst fomer studies focused on imaging by electron microscopy.

Our work and that of Ruben et al. as being complementary. We used several biophysical techniques in our work, most of which had also not been utilized frequently before to study $\beta 2$ GPI. Our findings differ from those of Ruben and colleagues and there are a number of possible reasons for this, apart from the use of different biophysical techniques. The source of $\beta 2$ GPI for the work of Ruben et al. was purified expressed $\beta 2$ GPI from cellular systems rather than from healthy or APS patient serum as shown in the previous studies ${ }^{5,29,40}$.

We used commercial $\beta 2 \mathrm{GPI}$, which is obtained from healthy human serum and therefore, a direct comparison of our results with Ruben's results is complicated.

Interestingly, a recent paper by Szabo et al..$^{41}$ also carried out imaging and binding studies of $\beta 2$ GPI purified by perchloric acid precipitation method which behaved similarly to $\beta 2 \mathrm{GPI}$ closed by $\mathrm{pH}$ gradient (confirmed by electron microscopy), whilst "opened" $\beta 2$ GPI (confirmed by electron microscopy) had significantly different effects in clotting assays. This contrasts with the work of Ruben et al. ${ }^{39}$ who show control $\beta 2$ GPI (unmodified) behaves similarly to the proven open expressed $\beta 2 \mathrm{GPI}$.

Similarly, Szabo et al. ${ }^{41}$ show that the open or closed $\beta 2$ GPI have comparable binding affinity to purified anti$\beta 2 \mathrm{GPI}$ antibodies, suggesting again that antibody binding might be structurally independent. This highlights the somewhat contrasting results within the field obtained by authors using diverse techniques.

Ioannou et al. used a different method to demonstrate free thiols in serum $\beta 2$ GPI from healthy subjects (not patients with APS) including binding to a streptavidin plate and using a murine anti- $\beta 2 \mathrm{GPI}^{29}$. Our work represents the first study of specifically reduced $\beta 2 \mathrm{GPI}$ and its binding to purified pathogenic anti-DI antibodies derived from patients with APS.

The effect of MPB cannot be discounted as it may give a steric effect on the opening of the molecule and it may also influence stability or purification. Biotin is known to interfere in circular dichroism spectroscopy below a wavelength of $215 \mathrm{~nm}$; as such we excluded this region from our spectra and focused on the 216-270 nm region. We also limited these effects by titrating the MPB concentration used and confirmed limited non-specific conjugation through LC-MS/MS analysis. Similarly, the presence of reduction in DI, which was previously published ${ }^{33,40,42}$, raises the possibility that the increased autoantibody binding seen is due to reduction in DI rather than DV. Although this is difficult to refute totally, the biotin quantification assay showed only one thiol per molecule and LC-MS/MS showed that the majority of molecules were labelled in the C-terminal peptide (Cys326). Therefore, the major change in antibody-binding we observed is most unlikely to be due to reduction in DI.

As discussed, the combination of LC-MS/MS and biotin quantification suggests that our system specifically labels the terminal disulfide Cys288/Cys326 of $\beta 2$ GPI. Labelling of the single thiol rather than both the thiols of the terminal disulfide may be due to the lack of solvent accessibility for Cys288, the partner of Cys326 (the C-terminal thiol). It should be highlighted, however, that the LC-MS/MS and biotin quantification cannot discount heterogeneity of the sample and thus, the inclusion of multiple labelled species and unlabelled species in minor amounts.

Molecular dynamics simulations showed no significant conformational shift upon reduction, which is consistent with our other data. Interestingly, however, a hydrogen bond near the terminal disulfide stabilized the circular form of $\beta 2 \mathrm{GPI}$. This is partially confirmed by reduced $\beta 2 \mathrm{GPI}$ having an increased flexibility of the loop regions close to the bond that allows for the disruption of this hydrogen bond. Nevertheless, further experimental work for validation is required.

Interestingly, our data suggest that, instead of a simple dichotomy between open and closed structures, the structural spectrum of $\beta 2$ GPI may be more subtle with ranges of flexibility.

Although DLS shows significant structural shifts from the non-modified protein, size measurements might be influenced by addition of MPB ligand which alters the electrophoretic properties of the protein. In addition, particle sizing methods can describe accurately spherical particles, but are limited regarding the size determination of non-spherical particles. A change in the lenght of non-spherical particles will impact the size, while a change in the diameter will be hard to detect (the diffusion will be barely influenced). DLS results suggest a more elongated character in reduced $\beta 2 \mathrm{GPI}$, as the hydrodynamic radius is smaller. This is consistent with the higher number of molecules with aspect ratio $>3$ in the AFM results.

CD spectroscopy results may confirm our theory for increased flexibility. However, due to HBS buffer interferences and strong interference of the MPB ligand (at least at wavelength below $215 \mathrm{~nm}$ ) in the CD spectra, it is difficult to assess whether the differences in the CD spectra of the untreated and reduced $\beta 2 \mathrm{GPI}$ are significant or not.

The striking change in activity towards APS autoantibodies between the reduced and untreated protein species could also be explained by increased flexibility in DV post-reduction, allowing the R39-R43 epitope in DI to become accessible in the fluid phase despite the molecule presenting an overall circular conformation.

Our study attempts at thoroughly associating the structure of reduced $\beta 2$ GPI with its function. The results show a clear change in binding to anti-DI from patients with APS after reduction, potentially explained by increased flexibility in DV. This has significant implications for how these pathogenic antibodies may respond in sites of oxidative stress where $\beta 2 \mathrm{GPI}$ is structurally flexible and thus more readily able to bind anti-DI antibodies. Through the use of structural techniques we refute several paradigms within the field including the idea that oxidized $\beta 2$ GPI must be linear. This refutation may have wide ranging implications for both our understanding 
of the molecule and, given the alteration in antibody binding, the pathogenesis of APS itself. Further studies are required to elucidate the relationship between function and structure in reduced $\beta 2 \mathrm{GPI}$.

\section{Methods}

Ethics. This study was carried out in accordance with recommendations of London Hampstead Research Ethics Committee Ref No 12/LO/0373 with written informed consent from all subjects in conformity with the Declaration of Helsinki. The experimental protocol was approved by the London Hampstead Research Ethics Committee Ref No 12/LO/0373.

Reagents. Unless otherwise stated, all chemicals were purchased from Sigma. Human plasma $\beta 2 \mathrm{GPI}$ was bought from Enzyme Research Laboratories. Protein concentration was determined using a bicinchoninic acid assay.

Ellman's reagent assay. To determine free thiol concentration, $35 \mu \mathrm{M}$ untreated $\beta 2 \mathrm{GPI}$ and samples for a cysteine hydrochloride monohydrate standard curve $(0-1.5 \mathrm{mM})$ were prepared in reaction buffer $(100 \mathrm{mM}$ sodium phosphate, $1 \mathrm{mM}$ EDTA, pH 8.0). $4 \mathrm{mg} / \mathrm{mL}$ Ellman's reagent (Thermo Fisher, Darmstadt, Germany) was also dissolved in reaction buffer. A mixture consisting of $200 \mu \mathrm{L}$ reaction buffer, $4 \mu \mathrm{L}$ Ellman's reagent and 20 $\mu \mathrm{L}$ sample or cysteine standard was added in a 96 well plate (Sarstedt, Nümbrecht, Germany) and incubated for $15 \mathrm{~min}$ at room temperature (RT) followed by absorption measurement at $412 \mathrm{~nm}$.

SDS-PAGE and western blot analysis. For SDS-PAGE, samples were treated under non-reducing conditions and loaded to $4-12 \%$ gradient bis-Tris SDS gels (Thermo Fisher). Gels were stained with SimplyBlue SafeStain (Thermo Fisher) or used for semidry Western blot transfer (30 min, $15 \mathrm{~V})$ to a nitrocellulose membrane (Amersham Protran $0.2 \mu \mathrm{m} \mathrm{NC)}$ ). Membrane was blocked with $5 \%$ milk powder in TBS (50 mM Tris, $0.15 \mathrm{M}$ $\mathrm{NaCl}, \mathrm{pH} 7.4$ ) containing $0.05 \%$ Tween 20 (TBST) for $1 \mathrm{~h}$ at RT and washed three times before incubation for $30 \mathrm{~min}$ at RT with peroxidase-conjugated streptavidin (Jackson ImmunoResearch) in 5\% milk powder/TBST. After three additional washing steps, the membrane was stained with peroxidase substrate (West Pico Chemiluminescent Substrate, Thermo Fisher).

$\beta 2$ GPI reduction. All reaction steps were performed under argon atmosphere at $25^{\circ} \mathrm{C} .50 \mu \mathrm{M}$ recombinant human thioredoxin-1 (TRX-1) was reduced by $50 \mu \mathrm{M}$ TCEP (Tris(2-carboxyethyl)phosphine hydrochloride) in a total volume of $100 \mu \mathrm{L}$ Hepes-buffered saline (HBS; $20 \mathrm{mM}$ Hepes, $140 \mathrm{mM} \mathrm{NaCl}, \mathrm{pH}$ 7.4) for $1 \mathrm{~h}$ with mild shaking. $900 \mu \mathrm{L}$ HBS buffer and $0.2 \mu \mathrm{M} \beta 2 \mathrm{GPI}$ were added and incubated for $1 \mathrm{~h}$ at with mild shaking. $15 \mu \mathrm{M}$ MPB (N ${ }^{a}$-(3-maleimidylpropionyl)biocytin; Thermo Fisher) were added for 15 min with mild shaking to label free thiols. Finally, $200 \mu \mathrm{M}$ reduced glutathione (GSH; Thermo Fisher) was used to quench unbound MPB (15 min). For purification, the reaction mixture was first volume-reduced by a $10 \mathrm{kDa}$ cut-off centrifugal filter unit (Merck) at $2500 \times g$, followed by size exclusion chromatography (SEC) with a Superdex 75 Increase column (GE Healthcare) with a flow rate of $0.25 \mathrm{~mL} / \mathrm{min}$ in HBS buffer. $\beta 2 \mathrm{GPI}$ main peak SEC fractions were pooled and concentrated using centrifugal filters with a cut-off of $10 \mathrm{kDa}$ (Merck) at $4000 \times g$.

LC-MS/MS sample preparation and analysis. For analysis of peptides of MPB labelled $\beta 2 \mathrm{GPI}, 4 \mu \mathrm{g}$ of total protein from each sample were mixed with $20 \mathrm{mM}$ ammonium bicarbonate, trypsin (Promega) was added at a ratio of 1:25 and samples were incubated overnight at $37^{\circ} \mathrm{C}$. Tryptic digestion was stopped through addition of acetic acid to a final concentration of $1 \%$ followed by desalting (ZipTip- $\mu$ C18 tips, Merck-Millipore). Extracts were concentrated by evaporation under vacuum and subsequently resolved in $0.1 \%$ acetic acid, $2 \%$ acetonitrile (ACN). LC-MS/MS analysis was performed as described previously ${ }^{43}$ with minor modifications. Chromatographic separation of tryptic peptides was achieved on a reverse phase nano-Acquity UPLC column $(1.7 \mu \mathrm{m}, 100 \mu \mathrm{m}$ i.d. $\times 100 \mathrm{~mm}$, Waters $\mathrm{GmbH})$ using a 50 min non-linear gradient from 2 to $60 \% \mathrm{ACN}$ in $0.1 \%$ acetic acid (flow rate: $400 \mathrm{~nL} / \mathrm{min}$ ). The nano-LC column was interfaced using electro-spray-ionization (ESI) to a LTQ-Orbitrap Velos mass spectrometer (Thermo Scientific). Precursor ions of a mass over charge range $(\mathrm{m} / \mathrm{z})$ 325-1525 ( $\mathrm{r}=30.000)$ were subjected to data dependent MS/MS fragmentation of Top-20 peaks in the ion trap at a collision induced energy (CID) of 35\%. Repetitive MS/MS acquisition was avoided by setting dynamic exclusion of $60 \mathrm{~s}$ for already selected precursors.

Data analysis was carried out using Proteome Discoverer 2.2 software (Thermo Scientific). Peptides were identified by search against the Uniprot human database (rel. 2017_01) using the following settings: trypsin as cleavage enzyme, 2 missed cleavage, oxidation on methionine, and biotin:Invitrogen-M1602 on cysteine residuals were selected as dynamic modifications. Only peptides identified with a false discovery rate (FDR) $<1 \%$ were used for further analysis. These peptides also demonstrated XCorr values $>2.0$.

Quantification of MPB labelling. To quantify the amount of MPB labelling per $\beta 2$ GPI molecule, a biotin quantification assay (colorimetric biotin assay kit, Sigma) was applied. $180 \mu \mathrm{L}$ of a $4^{\prime}$-hydroxyazobenzene2 -carboxylic acid (HABA)/avidin assay mixture and $20 \mu \mathrm{L}$ of $9-14 \mu \mathrm{M}$ reduced $\beta 2 \mathrm{GPI}$ sample, $20 \mu \mathrm{L}$ HBS buffer (negative control), or $20 \mu \mathrm{L}$ of $100 \mu \mathrm{M}$ biotin in ddH2O (positive control) were added to microtiter plate wells (Sarstedt). The reaction mixture was incubated for $5 \mathrm{~min}$ at RT with mild shaking $(150 \mathrm{rpm}$ ) in the dark and the absorbance was measured at $500 \mathrm{~nm}$. The biotin concentration was calculated according to the manufacturer's specifications using the extinctions coefficient of the HABA/avidin complex. 
Atomic force microscopy (AFM). AFM imaging of untreated as well as reduced $\beta 2$ GPI was executed on a Veeco NanoScope V with a JV-scanner (Bruker, Billerica, MA, USA). Images were recorded in AC/tapping mode in air using a PointProbe Plus AFM cantilever with a tip radius of curvature of $<10 \mathrm{~nm}$ and a spring constant of $42 \mathrm{~N} / \mathrm{m}$ (Nanosensors). A resonance frequency of approximately $330 \mathrm{kHz}$, as well as a sampling rate of $512 \times 512$ pixels with a scan rate of $1.0-1.5 \mathrm{~Hz}$, were applied. For AFM sample preparation, (untreated and reduced) $\beta 2 \mathrm{GPI}$ solutions were diluted to a concentration of $2 \mu \mathrm{g} / \mathrm{mL}$ in HBS buffer. $60 \mu \mathrm{L}$ of each sample was dropped on a freshly cleaved mica sheet (Electron Microscopy Sciences) and incubated for 5 min to allow protein adsorption. The excess solution was carefully removed from the edge of the mica sheet with a lint-free paper and the unbound protein was taken away from the surface by soaking the mica sheet in deionized water for $30 \mathrm{~s}$. Further, the water was removed with a lint-free paper and mica sheets were dried in a laminar flow box for $30 \mathrm{~min}$.

AFM image analysis. Analysis of $\beta 2$ GPI conformation from AFM imaging was done as we previously described ${ }^{26}$. Briefly, the aspect ratio $R$ (length/width) of single, flatly adsorbed $\beta 2$ GPI particles was calculated from AFM images. $\beta 2 \mathrm{GPI}$ in closed conformation were identified by an aspect ratio between $R=1$ and $R=3$. In contrast, $\beta 2 \mathrm{GPI}$ in open conformation led to a typical aspect ratio between $R=3$ and $R=10$. Hence, a threshold value of $R=3$ was chosen to distinguish between $\beta 2 \mathrm{GPI}$ in open and closed conformation in automated script analysis. $R$ value data for each analyzed $\beta 2$ GPI particle were represented as box plots. The quantiles of the box refer to 25 and $75 \%$, whereas the whisker includes minimum and maximum of the population. The percentage values of closed and open protein conformation were determined from the total number of analyzed particles.

Circular dichroism (CD) spectroscopy. CD measurements in the far-UV (215-270 nm) were carried out with a Chirascan $\mathrm{CD}$ spectrometer (Applied Photophysics) at $25^{\circ} \mathrm{C}$ using a protein concentration of $1 \mu \mathrm{M}$ in $\mathrm{HBS}$ buffer in $5 \mathrm{~mm}$ path length cuvettes. CD spectra acquired with a bandwidth of $1.0 \mathrm{~nm}$, a scanning speed of $15 \mathrm{~nm} /$ min and five repetitions were blank corrected and the wavelength dependent mean residue delta epsilon (MRDE) was calculated to normalize data for concentration, number of amino acids and path length of the cuvette.

Dynamic light scattering (DLS). DLS measurements were carried out in HBS buffer using a Zetasizer Nano-ZS (Malvern Instruments). $120 \mu \mathrm{L}$ of sample (6 $\mu \mathrm{M} \beta 2 \mathrm{GPI})$ previously vacuum-degassed for $20 \mathrm{~min}$ at $25^{\circ} \mathrm{C}$ were transferred to $10 \mathrm{~mm}$ path length cuvettes, equilibrated to $25^{\circ} \mathrm{C}$ for $5 \mathrm{~min}$ and measured 10 times (20 runs with run duration of $10 \mathrm{~s}$ each) at a detector angle of $173^{\circ}$. Hydrodynamic diameter $\left(\mathrm{D}_{\mathrm{H}}\right)$ data, using refractive index of 1.45 and absorption of 0.001 with standard solvent parameters as referred to water, was analyzed with Zetasizer 7.11 software was displayed as intensity $v s$. diameter size plot normalized to $100 \%$ intensity.

Purification of anti-DI IgG. Antibodies were purified as previously described ${ }^{20}$. Briefly, $50 \mu \mathrm{L}$ of $1 \mathrm{mg} / \mathrm{mL}$ Domain I (DI) was immobilized on nickel beads $(100 \mu \mathrm{L})$ for $30 \mathrm{~min}$ at RT on a rotating plate. Patient serum $(300 \mu \mathrm{L})$ was added (diluted 1:1 in $100 \mathrm{mM}$ sodium phosphate buffer saline (PBS), pH 7.4) and left to bind for $1 \mathrm{~h}$ rotating. Beads were washed with $100 \mathrm{mM}$ sodium phosphate buffer $(3 \times 1 \mathrm{~mL})$ and bound protein was eluted using $0.1 \mathrm{M}$ glycine ( $\mathrm{pH}$ 2.3). The eluate was dialysed against $100 \mathrm{mM}$ sodium phosphate, $150 \mathrm{mM} \mathrm{NaCl}, \mathrm{pH} 7.2$ and purified across a protein G column (GE Healthcare) before elution in $0.1 \mathrm{M}$ Glycine ( $\mathrm{pH} 2.3$ ) and dialysis to PBS for quantification. All patients were triple positive (a severe phenotype with exacerbated disease).

ELISA. ELISA procedure was adapted according to Agar et al. ${ }^{5}$. A microtiter plate (MaxiSorp Nunc-Immuno, Thermo Fisher) was coated with $100 \mu \mathrm{L} /$ well of either $5 \mu \mathrm{g} / \mathrm{mL}$ anti-DI antibody or $50 \mathrm{mM}$ carbonate buffer, $\mathrm{pH}$ 9.6 at $4{ }^{\circ} \mathrm{C}$ overnight. The plate was washed with TBS containing $0.1 \%$ Tween 20 and blocked with $200 \mu \mathrm{L} /$ well $3 \%$ bovine serum albumin (BSA) in TBS for $2 \mathrm{~h}$ at $37^{\circ} \mathrm{C}$. After washing the plate three times, $0,1,2$, or $5 \mu \mathrm{g} / \mathrm{mL}$ $\beta 2$ GPI species were incubated in $0.3 \%$ BSA/TBS $(100 \mu \mathrm{L} /$ well $)$ for $1 \mathrm{~h}$ at RT. The plate was washed three times and $100 \mu \mathrm{L}$ per well of peroxidase-conjugated anti- $\beta 2 \mathrm{GPI}$ antibody at $1 \mu \mathrm{g} / \mathrm{mL}$ (Affinity Biologicals) diluted in $0.3 \% \mathrm{BSA} / \mathrm{TBS}$ was added for $1 \mathrm{~h}$ at RT. After three final washing steps, $100 \mu \mathrm{L} /$ well TMB (BD Biosciences) was added, reaction was stopped by $100 \mu \mathrm{L} 1 \mathrm{~N} \mathrm{H}_{2} \mathrm{SO}_{4}$ after $15 \mathrm{~min}$ and absorption measured at $450 \mathrm{~nm}$. Statistical significance was derived by T-Tests and ANOVA using Graphpad Prism 8.

Control ELISA. Microtiter plates were coated with $50 \mu \mathrm{L} /$ well of 2, 5, or $10 \mu \mathrm{g} / \mathrm{mL} \beta 2 \mathrm{GPI}$ (either untreated or reduced) in TBS for $1 \mathrm{~h}$ at $37^{\circ} \mathrm{C}$. TBS alone was coated as a negative control. Plates were washed three times and blocked with $200 \mu \mathrm{L} /$ well $3 \%$ BSA/TBS for $2 \mathrm{~h}$ at $37^{\circ} \mathrm{C}$. The plate was washed again three times, before addition of $50 \mu \mathrm{L} /$ well $1 \mu \mathrm{g} / \mathrm{mL}$ peroxidase-conjugated anti- $\beta 2 \mathrm{GPI}$ antibody in $0.3 \%$ BSA/TBS for $1 \mathrm{~h}$ at RT. After three final washing steps, substrate was applied and absorption was measured at $450 \mathrm{~nm}$.

Modelling. To supplement the results of the structural experiments we carried out computer simulations of the effect of reduction at Cys288/Cys326 on the structure of $\beta 2$ GPI. Simulations were performed with GROMACS 5.1 software package ${ }^{44}$ using the AMBER99SB-ildn force field ${ }^{45}$. By increasing the hydrogen mass to 4 m.u. and reducing the corresponding heavy atom mass by the same amount (hydrogen mass repartitioning ${ }^{46}$, the verlet integrator ${ }^{47}$ was applied only every 7 femtoseconds. The explicit water was represented by the TIP3P model ${ }^{48}$ and carbohydrates with the Glycam force field ${ }^{49}$. Periodic boundary conditions were used together with the removal of the overall center of mass motion. Intermolecular Coulomb and van der Waals interactions were considered using cutoffs of $1.0 \mathrm{~nm}$ with switching functions at $0.9 \mathrm{~nm}$. Long-range electrostatic interactions were calculated with the PME method ${ }^{50}$ using a grid spacing of $1.2 \mathrm{~nm}$. All bonds were constraint to the optimal distance with the LINCS algorithm ${ }^{51}$. Temperature was controlled separately for protein and non-protein to $300 \mathrm{~K}$ every 100 
femtoseconds using a modified v-rescale thermostat ${ }^{52}$. A Parrinello-Rahman barostat ${ }^{53}$ adjusted the pressure to 1 bar every 12 picoseconds. All simulations were visualized with VMD 1.9.211 $1^{54}$.

Received: 10 October 2020; Accepted: 10 February 2021

Published online: 25 February 2021

\section{References}

1. Schwarzenbacher, R. et al. Crystal structure of human $\beta 2$-glycoprotein I: Implications for phospholipid binding and the antiphospholipid syndrome. EMBO J. 18, 6228-6239 (1999).

2. Baerenfaenger, M. \& Meyer, B. Simultaneous characterization of SNPs and N-glycans from multiple glycosylation sites of intact ß-2-glycoprotein-1 (B2GP1) by ESI-qTOF-MS. Biochim. Biophys. Acta Proteins Proteom. 1867, 556-564 (2019).

3. Kondo, A. et al. Glycopeptide profiling of beta-2-glycoprotein I by mass spectrometry reveals attenuated sialylation in patients with antiphospholipid syndrome. J. Proteom. 73, 123-133 (2009).

4. Gambino, R., Ruiu, G., Pagano, G. \& Cassader, M. Study of the glycosylation of apolipoprotein H. Chem. Phys. Lipids 103, 161-174 (1999).

5. Agar, C. et al. Two conformations: Implications for antigen recognition in the antiphospholipid syndrome. Blood 116, 1336-1343 (2010).

6. Bouma, B. et al. Adhesion mechanism of human beta 2-glycoprotein I to phospholipids based on its crystal structure. EMBO J. 18, 5166-5174 (1999).

7. de Laat, B. \& de Groot, P. G. Autoantibodies directed against domain I of Beta2-glycoprotein I. Curr. Rheumatol. Rep. 13, 70-76 (2011).

8. Pelkmans, L. \& de Laat, B. Antibodies against domain I of $\beta 2$-glycoprotein I: the one and only? Lupus 21, 769-772 (2012).

9. de Groot, P. G. \& Urbanus, R. T. The significance of autoantibodies against $\beta 2$-glycoprotein I. Blood 120, 266-274 (2012).

10. de Laat, B. et al. The association between circulating antibodies against domain I of beta2-glycoprotein I and thrombosis: An international multicenter study. J. Thromb. Haemost. 7, 1767-1773 (2009).

11. Di Simone, N. et al. Pathogenic role of anti- $\beta 2$-glycoprotein I antibodies on human placenta: functional effects related to implantation and roles of heparin. Hum. Reprod. Update 13, 189-196 (2007).

12. Bas de Laat, H., Derksen, R. H. \& de Groot, P. G. $\beta 2$-Glycoprotein I, the playmaker of the antiphospholipid syndrome. Clin. Immunol. 112, 161-168 (2004).

13. Pericleous, C. et al. Measuring IgA anti- $\beta 2$-glycoprotein I and IgG/IgA anti-Domain I antibodies adds value to current serological assays for the antiphospholipid syndrome. PLOS ONE 11, e0156407 (2016).

14. Duarte-Garcia, A. et al. The epidemiology of antiphospholipid syndrome: A population-based study. Arthritis Rheumatol. 71, 1545-1552 (2019).

15. Ioannou, Y. \& Rahman, A. Domain I of $\beta 2$-glycoprotein I: Its role as an epitope and the potential to be developed as a specific target for the treatment of the antiphospholipid syndrome. Lupus 19, 400-405 (2010).

16. Meroni, P. L. Anti-beta-2 glycoprotein I epitope specificity: From experimental models to diagnostic tools. Lupus 25, 905-910 (2016).

17. Chighizola, C. B. et al. Beyond thrombosis: Anti- $\beta 2$ GPI domain 1 antibodies identify late pregnancy morbidity in anti-phospholipid syndrome. J. Autoimmun. 90, 76-83 (2018).

18. Reddel, S. W., Wang, Y. X., Sheng, Y. H. \& Krilis, S. A. Epitope studies with anti- $\beta 2$-glycoprotein I antibodies from autoantibody and immunized sources. J. Autoimmun. 15, 91-96 (2000).

19. Liu, T. et al. Anti- $\beta 2$ GPI domain 1 antibodies stratify high risk of thrombosis and late pregnancy morbidity in a large cohort of Chinese patients with antiphospholipid syndrome. Thromb. Res. 185, 142-149 (2020).

20. Pericleous, C. et al. Proof-of-concept study demonstrating the pathogenicity of affinity-purified IgG antibodies directed to domain I of 2-glycoprotein I in a mouse model of anti-phospholipid antibody-induced thrombosis. Rheumatology (Oxford) 54, 722-727 (2015).

21. Iwaniec, T., Kaczor, M. P., Celinska-Lowenhoff, M., Polanski, S. \& Musial, J. Clinical significance of anti-domain $1 \beta 2$-glycoprotein I antibodies in antiphospholipid syndrome. Thromb. Res. 153, 90-94 (2017).

22. de Laat, B. et al. Immune responses against domain I of $\beta 2$-glycoprotein I are driven by conformational changes: Domain I of $\beta 2$-glycoprotein I harbors a cryptic immunogenic epitope. Arthritis Rheum. 63, 3960-3968 (2011).

23. McDonnell, T. et al. The role of beta-2-glycoprotein I in health and disease associating structure with function: More than just APS. Blood Rev. 39, 100610 (2020).

24. Agar, C. et al. ß2-Glycoprotein I: A novel component of innate immunity. Blood 117, 6939-6947 (2011).

25. Leu, S. J. et al. Structural and functional characterization of $\beta 2$-glycoprotein I domain 1 in anti-melanoma cell migration. Cancer Sci. 110, 1974-1986 (2019).

26. Buchholz, I., Nestler, P., Köppen, S. \& Delcea, M. Lysine residues control the conformational dynamics of beta 2-glycoprotein I. PCCP 20, 26819-26829 (2018)

27. El-Assaad, F., Krilis, S. A. \& Giannakopoulos, B. Posttranslational forms of beta 2-glycoprotein I in the pathogenesis of the antiphospholipid syndrome. Thromb. J. 14, 20 (2016).

28. Schmidt, B., Ho, L. \& Hogg, P. J. Allosteric disulfide bonds. Biochemistry 45, 7429-7433 (2006).

29. Ioannou, Y. et al. Naturally occurring free thiols within $\beta 2$-glycoprotein I in vivo: Nitrosylation, redox modification by endothelial cells, and regulation of oxidative stress-induced cell injury. Blood 116, 1961-1970 (2010).

30. Lopez-Pedrera, C. et al. Oxidative stress in the pathogenesis of atherothrombosis associated with anti-phospholipid syndrome and systemic lupus erythematosus: New therapeutic approaches. Rheumatology (Oxford) 55, 2096-2108 (2016).

31. Lopez, L. R., Simpson, D. F., Hurley, B. L. \& Matsuura, E. OxLDL/ß2GPI complexes and autoantibodies in patients with systemic lupus erythematosus, systemic sclerosis, and antiphospholipid syndrome: Pathogenic implications for vascular involvement. Ann. N. Y. Acad. Sci. 1051, 313-322 (2005).

32. Raimondo, M. G. et al. Oxidation of $\beta 2$-glycoprotein I associates with IgG antibodies to domain I in patients with antiphospholipid syndrome. PLoS ONE 12, e0186513 (2017).

33. Passam, F. H., Giannakopoulos, B., Mirarabshahi, P. \& Krilis, S. A. Molecular pathophysiology of the antiphospholipid syndrome: The role of oxidative post-translational modification of beta 2 glycoprotein I. J. Thromb. Haemost. 9(Suppl 1), 275-282 (2011).

34. Passam, F. H. et al. Beta 2 glycoprotein I is a substrate of thiol oxidoreductases. Blood 116, 1995-1997 (2010).

35. Acquasaliente, L. et al. Molecular mapping of $\alpha$-thrombin $(\alpha \mathrm{T}) / \beta 2$-glycoprotein I ( $\beta 2 \mathrm{GpI})$ interaction reveals how $\beta 2 \mathrm{GpI}$ affects aT functions. Biochem. J. 473, 4629-4650 (2016).

36. Pengo, V. et al. Clinical course of high-risk patients diagnosed with antiphospholipid syndrome. J. Thromb. Haemost. 8, 237-242 (2010).

37. Pengo, V. et al. Rivaroxaban vs warfarin in high-risk patients with antiphospholipid syndrome. Blood 132, 1365-1371 (2018). 
38. Ioannou, Y. et al. Novel assays of thrombogenic pathogenicity in the antiphospholipid syndrome based on the detection of molecular oxidative modification of the major autoantigen 32 -glycoprotein I. Arthritis Rheum. 63, 2774-2782 (2011).

39. Ruben, E. et al. The J-elongated conformation of $\beta 2$-glycoprotein I predominates in solution: Implications for our understanding of antiphospholipid syndrome. J. Biol. Chem. 295, 10794-10806 (2020).

40. Passam, F. H. et al. Redox control of $\beta 2$-glycoprotein I-von Willebrand factor interaction by thioredoxin-1. J. Thromb. Haemost. 8, 1754-1762 (2010)

41. Szabo, G. et al. Distinct and overlapping effects of $\beta 2$-glycoprotein I conformational variants in ligand interactions and functional assays. J. Immunol. Methods 487, 112877 (2020).

42. Giannakopoulos, B. \& Krilis, S. A. The pathogenesis of the antiphospholipid syndrome. N. Engl. J. Med. 368, 1033-1044 (2013).

43. Kindt, F. et al. A novel assay to assess the effect of pharmaceutical compounds on the differentiation of podocytes: Screening assay of podocyte differentiation. Br. J. Pharmacol. 174, 163-176 (2017).

44. Abraham, M. J. et al. GROMACS: High performance molecular simulations through multi-level parallelism from laptops to supercomputers. SoftwareX 1-2, 19-25 (2015).

45. Lindorff-Larsen, K. et al. Improved side-chain torsion potentials for the Amber ff99SB protein force field. Proteins Struct. Funct. Bioinform. 78, 1950-1958 (2010)

46. Feenstra, K. A., Hess, B. \& Berendsen, H. J. C. Improving efficiency of large time-scale molecular dynamics simulations of hydrogenrich systems. J. Comput. Chem. 20, 786-798 (1999).

47. Frenkel, D. \& Smit, B. Introduction. In Understanding Molecular Simulation 2nd edn (eds Frenkel, D. \& Smit, B.) 1-6 (Academic Press, San Diego, 2002).

48. Jorgensen, W. L., Chandrasekhar, J., Madura, J. D., Impey, R. W. \& Klein, M. L. Comparison of simple potential functions for simulating liquid water. J. Chem. Phys. 79, 926-935 (1983).

49. Kirschner, K. N. et al. GLYCAM06: A generalizable biomolecular force field. Carbohydrates: GLYCAM06. J. Comput. Chem. 29, 622-655 (2008).

50. Ewald, P. P. Die Berechnung optischer und elektrostatischer Gitterpotentiale. Ann. Phys. 369, 253-287 (1921).

51. Hess, B., Bekker, H., Berendsen, H. J. C. \& Fraaije, J. G. E. M. LINCS: A linear constraint solver for molecular simulations. J. Comput. Chem. 18, 1463-1472 (1997).

52. Bussi, G., Donadio, D. \& Parrinello, M. Canonical sampling through velocity rescaling. J. Chem. Phys. 126, 014101 (2007).

53. Parrinello, M. \& Rahman, A. Polymorphic transitions in single crystals: A new molecular dynamics method. J. Appl. Phys. 52, 7182-7190 (1981).

54. Humphrey, W., Dalke, A. \& Schulten, K. VMD: Visual molecular dynamics. J. Mol. Graph. 14, 33-38 (1996).

\section{Acknowledgements}

We acknowledge Felix Nagel for his help in preparing the manuscript and fruitful discussions. This work was financially supported by the European Research Council (ERC) Starting Grant 'PredicTOOL' (637877) to MD. Support by the UCL Global Engagement Office (GEO) Sea and Current Grant 501084 is also acknowledged. Funding was also given by the MRF (MRF-057-0004-RG-MCDO-C0800)), Lupus UK (1579346) and the MRC (Grant MR/P017371/1).

\section{Author contributions}

I.B. designed the experiments, carried out most of the experiments, analyzed and interpreted the results, wrote the manuscript. T.M. designed the experiments, carried out the antibody purification, analyzed and interpreted the results, wrote the manuscript. P.N. analyzed and discussed the AFM data. S.T. carried out the AFM experiments. M.K. carried out the MDS studies. A.R. assisted on the initial reduction protocol establishment. V.M.R. advised on the initial project plan. F.S. and E.H. helped with the design and interpretation of the MS experiments. J.L.T.H. discussed the AFM data. A.R. developed the study concept, provided the patient samples, interpreted the data and wrote the manuscript. M.D. developed the study concept and interpreted the data and wrote the manuscript. All authors reviewed and approved the final version of the manuscript.

\section{Funding}

Open Access funding enabled and organized by Projekt DEAL.

\section{Competing interests}

The authors declare no competing interests.

\section{Additional information}

Supplementary Information The online version contains supplementary material available at https://doi.org/ 10.1038/s41598-021-84021-2.

Correspondence and requests for materials should be addressed to A.R. or M.D.

Reprints and permissions information is available at www.nature.com/reprints.

Publisher's note Springer Nature remains neutral with regard to jurisdictional claims in published maps and institutional affiliations.

\footnotetext{
(c) (i) Open Access This article is licensed under a Creative Commons Attribution 4.0 International cc) License, which permits use, sharing, adaptation, distribution and reproduction in any medium or format, as long as you give appropriate credit to the original author(s) and the source, provide a link to the Creative Commons licence, and indicate if changes were made. The images or other third party material in this article are included in the article's Creative Commons licence, unless indicated otherwise in a credit line to the material. If material is not included in the article's Creative Commons licence and your intended use is not permitted by statutory regulation or exceeds the permitted use, you will need to obtain permission directly from the copyright holder. To view a copy of this licence, visit http://creativecommons.org/licenses/by/4.0/.
}

(C) The Author(s) 2021 